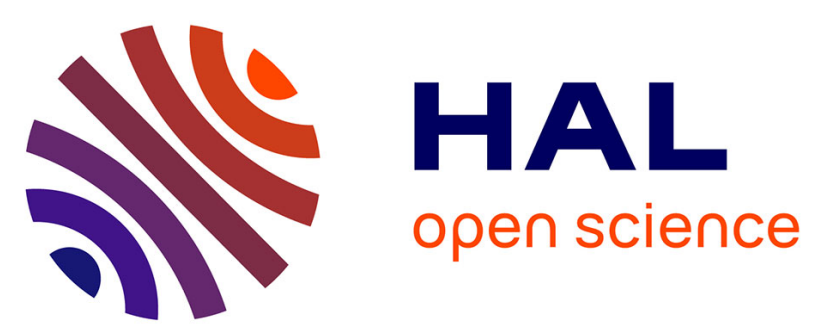

\title{
Tuning the nonlinear optical properties of BODIPYs by functionalization with dimethylaminostyryl substituents
}

\author{
Bohdan Kulyk, Said Taboukhat, Huriye Akdas-Kilig, Jean-Luc Fillaut,
} Miroslav Karpierz, Bouchta Sahraoui

\section{- To cite this version:}

Bohdan Kulyk, Said Taboukhat, Huriye Akdas-Kilig, Jean-Luc Fillaut, Miroslav Karpierz, et al.. Tuning the nonlinear optical properties of BODIPYs by functionalization with dimethylaminostyryl substituents. Dyes and Pigments, 2017, 137, pp.507-511. 10.1016/j.dyepig.2016.10.045 . hal-01438103

HAL Id: hal-01438103

https://hal-univ-rennes1.archives-ouvertes.fr/hal-01438103

Submitted on $13 \mathrm{Jul} 2017$

HAL is a multi-disciplinary open access archive for the deposit and dissemination of scientific research documents, whether they are published or not. The documents may come from teaching and research institutions in France or abroad, or from public or private research centers.
L'archive ouverte pluridisciplinaire HAL, est destinée au dépôt et à la diffusion de documents scientifiques de niveau recherche, publiés ou non, émanant des établissements d'enseignement et de recherche français ou étrangers, des laboratoires publics ou privés. 


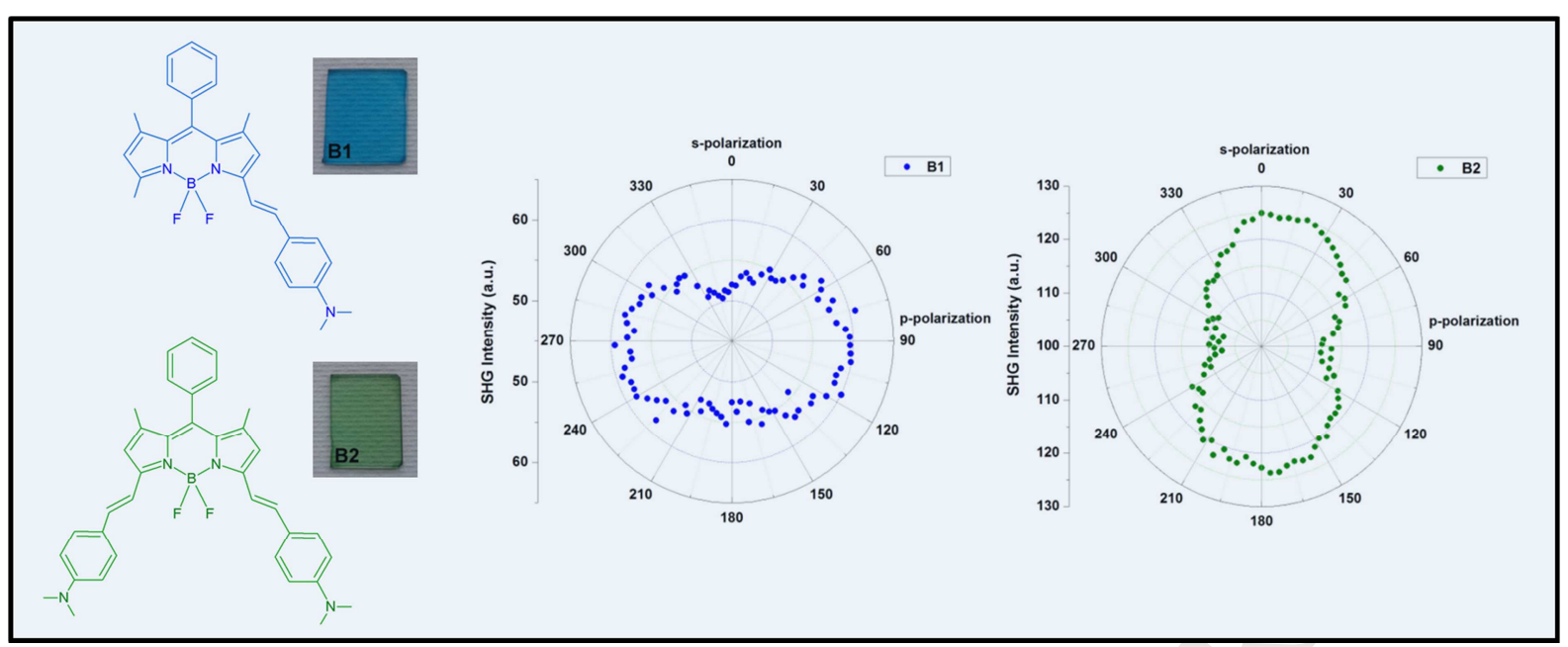




\section{Tuning the Nonlinear Optical Properties of BODIPYs by}

\section{Functionalization with Dimethylaminostyryl Substituents}

4 Bohdan Kulyk $^{\mathrm{a}, *}$, Said Taboukhat ${ }^{\mathrm{a}, \mathrm{b}}$, Huriye Akdas-Kilig ${ }^{\mathrm{c}}$, Jean-Luc Fillaut ${ }^{\mathrm{c}}$, Miroslav Karpierz ${ }^{\mathrm{d}}$,

$5 \quad$ Bouchta Sahraoui ${ }^{a}$,**

6

7 aniversity of Angers, MOLTECH-Anjou Laboratory, UMR CNRS 6200, 2 bd Lavoisier,

849045 Angers, France

9 baboratory of Bio-Geosciences and Materials Engineering, ENS, University Hassan II of

10 Casablanca, BP 50069, Casablanca, Morocco

$11{ }^{\mathrm{c}}$ Institut des Sciences Chimiques de Rennes, UMR CNRS 6226, University of Rennes 1,

12 263, avenue du General Leclerc, 35042 Rennes, France

$13{ }^{\mathrm{d}}$ Warsaw University of Technology, Faculty of Physics, Koszykowa 75, 00-662 Warsaw,

14 Poland

15

$16 *$ Corresponding author. University of Angers, MOLTECH-Anjou Laboratory, UMR

17 CNRS 6200, 2 bd Lavoisier, 49045 Angers, France

$18 * *$ Corresponding author. University of Angers, MOLTECH-Anjou Laboratory, UMR

19 CNRS 6200, 2 bd Lavoisier, 49045 Angers, France

20 E-mail addresses: bohdan_kulyk@yahoo.com (B. Kulyk), 


\section{Abstract}

Two difluoroboradiazaindacene (BODIPY) scaffolds with attached dimethylaminostyryl

Keywords: BODIPY dye, polymer composites, second/third harmonic generation, nonlinear susceptibility, hyperpolarizability substituents were synthesized. Guest-host polymeric films were produced by incorporating these chromophores into polymethylmethacrylate matrices. The second and third nonlinear optical properties of the resulting polymer composites were studied by means of the Maker fringe technique using a laser generating at $1064 \mathrm{~nm}$ with a $30 \mathrm{ps}$ pulse duration. The macroscopic and microscopic nonlinearities were found to be comparatively high and dependent on the number of dimethylaminostyryl substituents attached to BODIPY core. The development of integrated optics makes such nonlinear films of particular interest, since they can be used in the creation of efficient nonlinear devices.

(1)

\section{susceptibility, hyperpolarizability}

\section{Introduction}

In recent years, difluoroboradiazaindacene (BODIPY) unit has attracted much interest because of valuable spectroscopic and photophysical properties such as its high molar absorptivity coefficient [1], high fluorescence quantum yield [2,3] and good stability towards light and chemicals [4]. Recently many BODIPY derivatives have been synthesized and utilized as fluorescent dyes [5], chemosensors [6-10], polarity sensitive probe [11], light harvesting systems [12,13], and for laser applications [14] and photodynamic therapy [15,16]. 
This research work is devoted to nonlinear optical (NLO) investigations of BODIPY model

46 compounds by means of SHG and THG (second/third harmonic generation) techniques, which

47 allow the determination of second and third order NLO parameters. Until now a small set of

48 BODIPY derivatives have been investigated for nonlinear optics [17-23]. Recently, much

49 attention has been devoted to functionalized BODIPY dyes that exhibit two-photon absorption

50 (TPA) properties due to their related application in TPA imaging [24-26]. To this end, extending

51 conjugation and forming Intramolecular Charge Transfer (ICT) by means of appropriate electron

52 donor or acceptor groups are effective strategies for the synthesis of highly efficient two-photon

53 active BODIPY compounds. Thus, understanding how incorporating electron donor and/or

54 acceptor groups in the BODIPY architecture affect NLO response remains a challenge.

55 Our research work is focused on two BODIPY-based models which have one or two

56 dimethylaminostyryl branches, with high ICT strength from the dialkylamino-groups electron

57 donors to the electron acceptor boradipyrrin core. Interestingly, B1 and B2 models not only

58 present intrinsic "push-pull" character, but also differ by their inherent D-A (B1) and D-A-D (B2)

59 structures that potentially govern distinct second and third order optical nonlinearities. Recently,

60 we have shown that dimethylaminostyryl arms affect the nonlinear refraction and absorption

61 activity of the BODIPY structure [27]. Meanwhile, the SHG and THG study of

62 dimethylaminostyryl substituted BODIPY-based derivatives has not been done yet, so the results

63 of this investigation may extend the application range of the dye. The second and third nonlinear

64 optical properties of BODIPY-based materials, as guest-host polymeric films produced by

65 incorporating the chromophores into PMMA matrices, were studied by means of the Maker

66 fringe technique. The NLO susceptibilities and hyperpolarizabilities were extracted, which can

67 give a better understanding on the origin of NLO phenomena and the structure-property

68 relationships in BODIPY chromophores. 
69

70

71

72

73

74

75

76

77

78

79

80

81

82

83

84

85

\section{Experimental section}

\subsection{Materials}

The BODIPY models have been synthesized in good yield by the established Konoevenagel condensation of 3,5 dimethyl-BODIPY dyes with 4-dimethylaminobenzaldehyde, as it is previously described by Rurack [28] and Akkaya [29]. The structures of the resulting difluoroboradiazaindacene chromophores with dimethylaminostyryl substituents (namely B1 and B2) are shown in Fig. 1. The monocondensation was controlled by adjusting the mole ratio and stopping the reaction after few hours, while a larger excess of 4-dimethylaminobenzaldehyde and a longer reaction time increased the yield of by-condensed product B2 (Supporting Information).

a)

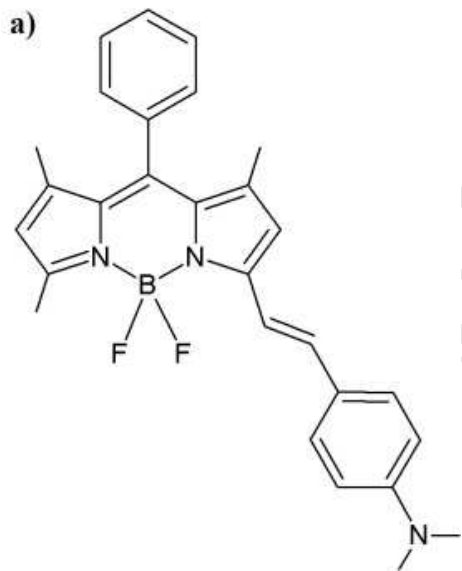

b)

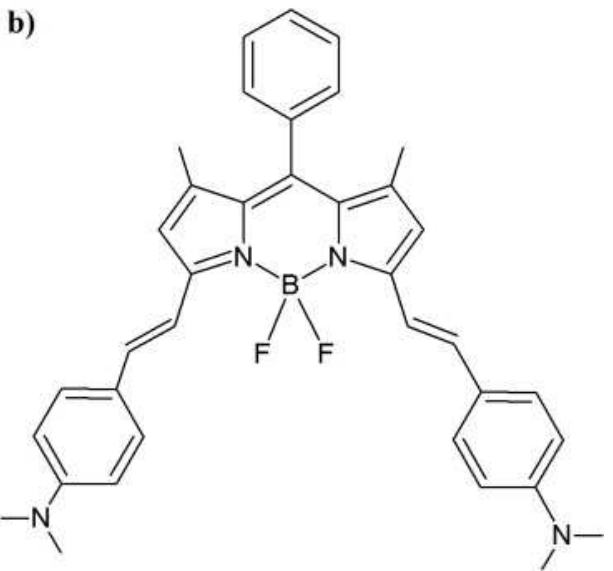

Fig. 1. Structures of the conjugated difluoroboradiazaindacene chromophores with dimethylaminostyryl substituents: a) B1; b) B2.

\subsection{Film fabrication}

For the polymeric film preparation a solution of PMMA (Sigma-Aldrich, Mw=350,000 $\mathrm{g} / \mathrm{mol}$ ) dissolved in 1,1,2-trichloroethane at concentration of $50 \mathrm{~g} / \mathrm{L}$ was used as a host system. 
86 The concentration of the compounds was $100 \mu \mathrm{mol}$ towards $1 \mathrm{~g}$ of PMMA. The glass plates were

87 washed in distilled water using ultrasonic bath, acetone, and ethanol and then dried. The solutions

88 were deposited on glass substrates using the spin-coater (SCS G3) at $1000 \mathrm{rpm}$. Obtained guest-

89 host polymer films were kept at room temperature during few days in order to eliminate any

90 remaining of solvent. The thickness of deposited films was measured with the profilometer

91 (Dektak 6M, Veeco) to be about $700 \mathrm{~nm}$.

92

93

94

95

96

97

98

99

100

101

102

103

104

105

106

107

\subsection{Optical absorption measurements}

Absorption spectra of the B1 and B2 films were measured by means of Lambda 950 UV/Vis/NIR spectrophotometer (PerkinElmer) in the range $300-1200 \mathrm{~nm}$. The pure PMMA film on glass plate was used on the way of reference beam in spectrophotometer for the measurements of absorption spectra of chromophores.

\subsection{SHG and THG measurements}

The phenomena of second and third harmonic generation (SHG and THG) may take place in a material the nonlinear polarization of which, caused by intensive laser irradiation, stimulates the coherent light emission at double and triple laser frequencies. The schematically energy-level diagram of SHG and THG processes is shown in Fig. 2a. SHG and THG measurements were performed by means of the rotational Maker fringe technique [30] in the transmission scheme for the $s$ - and $p$-polarized fundamental laser beams (Fig. 2b). A y-cut crystalline quartz plate has been used as a reference material for SHG measurements and fused silica plate for THG measurements. The output beam of a mode-locked Nd:YAG/YVO 4 laser (EKSPLA) generating at $\lambda=1064 \mathrm{~nm}$ with $30 \mathrm{ps}$ pulse duration and $10 \mathrm{~Hz}$ repetition rate was employed as a fundamental beam. The input energy of laser pulses was controlled by laser power/energy meter (LabMax 
108 TOP, COHERENT) to be $90 \mu \mathrm{J}$ for SHG and $150 \mu \mathrm{J}$ for THG measurements. The detailed setups

109 description can be found elsewhere [31].

110
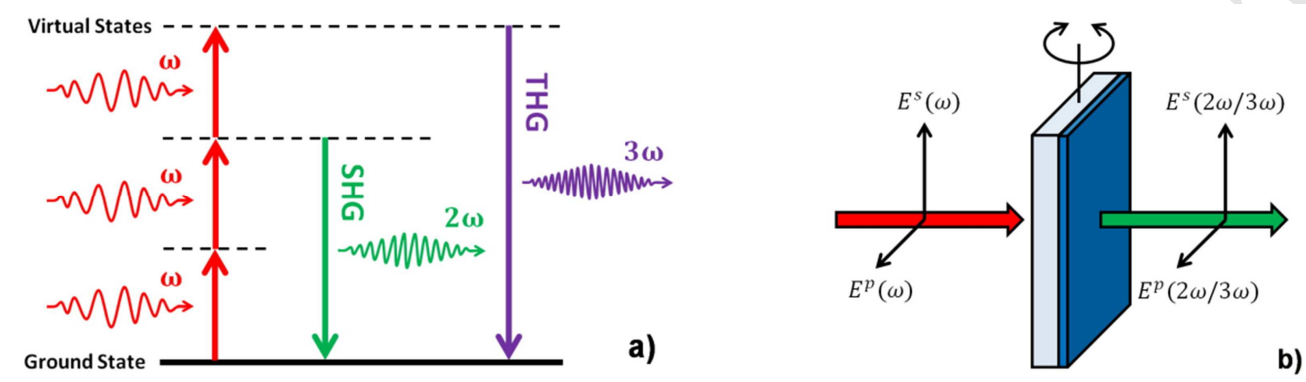

112 Fig. 2. a) Energy-level diagram of SHG and THG processes and b) the illustration of input-output

113 polarization scheme in Maker fringe experiment.

\section{Results and discussion}

\subsection{Optical absorption properties}

The absorption spectra of B1 and B2 compounds embedded in PMMA films at the

118 concentration $100 \mu \mathrm{mol} / \mathrm{g}$ are given in Fig. 3. As expected, attaching the electron donating

119 dimethylamino groups at the 3 and 5 positions, through a conjugated bridge, leads to considerable

120 shift of the absorption band towards the red region, by comparison with the parent BODIPY

121 compound [2, 20]: UV-Vis $\left(\mathrm{CH}_{2} \mathrm{Cl}_{2}\right) \lambda_{\max }($ abs. $)=598 \mathrm{~nm}(\mathrm{~B} 1), 692 \mathrm{~nm}$ (B2). The UV-Vis

122 absorption and fluorescent emission spectra of the THF solutions displayed the maximal 123 absorbance at $\lambda_{\max }($ abs. $)=601 \mathrm{~nm}(\mathrm{~B} 1), 694 \mathrm{~nm}$ (B2) with a molar extinction coefficient of about $12490,000 \mathrm{M}^{-1} \mathrm{~cm}^{-1}$ and the maximal emission at $\lambda_{\max }(\mathrm{em})=.643 \mathrm{~nm}(\mathrm{~B} 1), 722 \mathrm{~nm}$ (B2) with the

125 fluorescence quantum yield of 0.24 and 0.15 for B1 and B2, respectively [27]. Similarly, the 126 spectra of both films exhibit wide absorption bands with maximum at 605 and $703 \mathrm{~nm}$ for B1 and 
127 B2, respectively, which correspond to $S_{0} \rightarrow S_{1}\left(\pi-\pi^{*}\right)$ transitions. The short-wavelength vibronic

128 shoulder of these bands can be assigned to a $\mathrm{C}-\mathrm{H}$ out-of-plane vibration [32]. The absorption

129 peaks at the wavelength less than $500 \mathrm{~nm}$ could be attributed to a partially forbidden $\mathrm{S}_{0} \rightarrow \mathrm{S}_{2}(\pi-$

$\left.130 \pi^{*}\right)$ transitions in BODIPY core [33]. As the absorption spectra indicates, introduction of second

131 electron-donor dimethylaminostyryl group exerts almost $100 \mathrm{~nm}$ red-shift of absorption band

132 without considerable change of its intensity. At the wavelengths of more than $800 \mathrm{~nm}$ the

133 samples show high optical transparency.

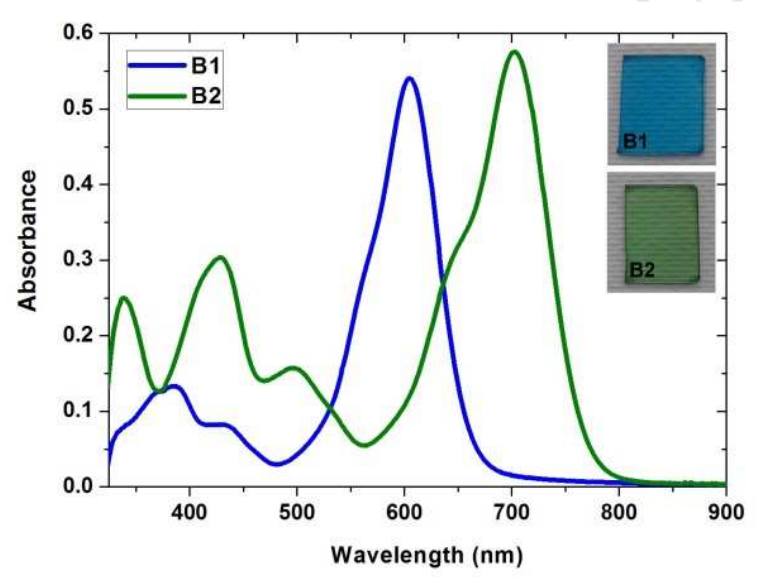

136 Fig. 3. UV-vis absorption spectra of the B1 and B2 compounds embedded in PMMA films. In 137 inset - the photos of the deposited films.

\subsection{SHG response study}

The rotational Maker fringe technique was implemented for the measurements of SHG in B1

141 and B2 guest-host polymeric films for $s$ - and $p$-polarized fundamental laser beam. Since the 142 dipole moments of the chromophores are initially randomly oriented within the film, the second 143 order nonlinear optical response of the film is negligible due to its macroscopic centrosymmetry.

144 In order to break the centrosymmetry and induce the uniaxial orientation of NLO chromophores 
145 in the polymeric films, provoking their manifestation of second order NLO behavior, the samples

146 were corona poled just before SHG measurements. The poling temperature was $95^{\circ} \mathrm{C}$, a little

147 lower than the glass transition temperature and applied electric field was $5 \mathrm{kV} / \mathrm{cm}$. In Fig. 4 the

148 dependences of the second harmonic intensity generated as the function of incident angle are

149 presented. The obtained angular dependencies are typical for induced $C_{\infty \infty v}$ symmetry view with a

150 maximum signal at $60^{\circ}-65^{\circ}$ and zero intensity at normal incidence of the fundamental beam. The

151 polarization of the second harmonic signal was found to be always $p$-polarized regardless of the

152 incident beam polarization due to the peculiarities of symmetry dependent second order 153 susceptibility tensor components.

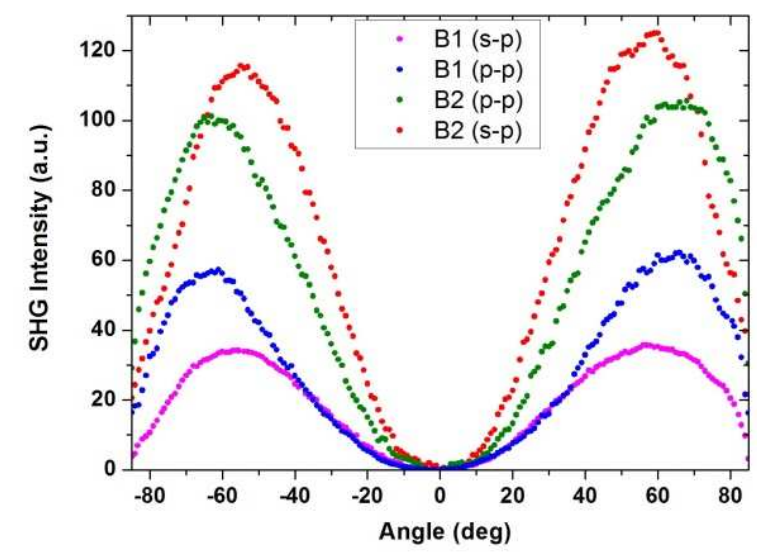

156 Fig. 4. SHG intensity as a function of incident angle in B1 and B2 guest-host films at the $s^{-}$, and $p$-polarized fundamental beam.

As it can be seen for $s-p$ or $p-p$ polarizations, the intensity of SHG from B2 compound which

160 has two dimethylaminostyryl units is almost two-to-three times higher than the intensity of B1

161 with one dimethylaminostyryl unit. In contrast to B1 film, the SHG response from B2 film for $s-p$

162 (input-output) polarization is higher than for $p-p$ polarization. For B1 the higher SHG signal can 
163 be obtained when the polarization of incident beam is close to parallel to its dipole moment or

164 direction between donor (D) amine group and acceptor (A) BODIPY core, meanwhile the higher

165 SHG signal in B2 can be obtained when the polarization of incident beam is rather perpendicular

166 to D-A direction. In the Fig. 5 the dependences of SHG intensity on polarization of incident beam

167 measured at $60^{\circ}$ are presented, which confirm the contrary directions in the molecules where

168 highest response occurs. The second order nonlinearity of BODIPY with attached

169 dimethylaminostyryl substituents is more efficient in D-A-D architecture (B2), in which two ICT

170 processes contribute to the NLO response, than in D-A (B1).
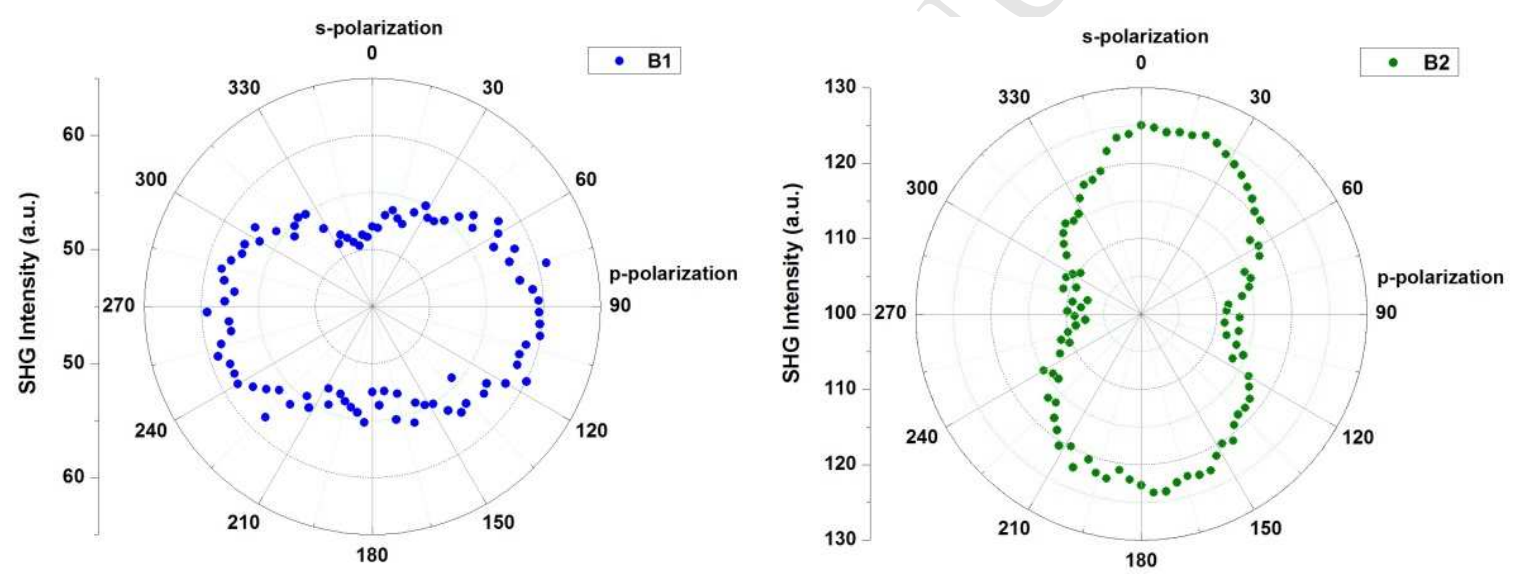

173 Fig. 5. The dependences of SHG intensity on incident beam polarization in B1 and B2 films.

Since the films have the thickness much less than their coherence length, the intensities of SHG from films were compared to that from a quartz plate and the quadratic NLO susceptibilities were calculated using following equation taking into account linear optical absorption [34]:

178

$$
\chi^{(2)}=\chi_{\text {Quartz }}^{(2)}\left(\frac{2}{\pi}\right)\left(\frac{l_{\text {Quartz }}^{\text {coh }}}{d}\right)\left(\frac{\frac{\alpha d}{2}}{1-e^{-\frac{\alpha d}{2}}}\right)\left(\frac{I^{2 \omega}}{I_{\text {Quartz }}^{2 \omega}}\right)^{1 / 2},
$$


181 where $\chi_{\text {Quartz }}^{(2)}$ is the quadratic susceptibility of quartz, $l_{\text {Quartz }}^{\text {coh }}$ is the coherent length of quartz, $\alpha$

182 is the absorption coefficient at doubled laser wavelength, $d$ is the film thickness, $I^{2 \omega}$ and $I_{\text {Quartz }}^{2 \omega}$

183 are the SHG intensities from the sample and quartz under the same conditions of measurement,

184 respectively. The obtained effective quadratic NLO susceptibilities are presented in Table 1. Here

185 again, the effective values of $\chi^{(2)}$ for B2 compound were found to be higher than that for B1 due

186 to the doubled amount of ICT which plays a crucial role in nonlinear behavior of a molecule. The

187 obtained values of quadratic susceptibility are quite high and comparable with those which we

188 have recently obtained for spin-deposited thin films of azobenzene-based push-pull compounds

189 [35]. Meanwhile, in the microscopic approach, the first hyperpolarisabilities of B1 and B2

190 extracted form $\chi^{(2)}$ assuming parallel orientations of dipole moment of molecules towards polar

191 axis induced by the poling field are found to be one order of magnitude lower than for other

192 BODIPY-based derivatives [19].

193

194 Table 1. The values of $\chi^{(2)}$ eff, $\chi^{(3)}$ elec, $\beta_{\text {eff }}$ and $\gamma_{\text {elec }}$ obtained for B1 and B2.

\begin{tabular}{cccccc}
\hline Compound & $\begin{array}{c}\chi_{\text {eff, }}^{(2)} \\
\left(\mathrm{pm} \mathrm{V}^{-1}\right)\end{array}$ & $\begin{array}{c}\chi_{\text {eff, }}^{(2)}\left(\mathrm{pm} \mathrm{V}^{-1}\right) \\
\left(10^{-38} \mathrm{~m}^{4} \mathrm{~V}^{-1}\right)\end{array}$ & $\begin{array}{c}\chi_{\text {efec }}^{(3)}, s-s \\
\left(10^{-20} \mathrm{~m}^{2} \mathrm{~V}^{-2}\right)\end{array}$ & $\begin{array}{c}\gamma_{\text {elec }}, \\
\left(10^{-47} \mathrm{~m}^{5} \mathrm{~V}^{-2}\right)\end{array}$ \\
\hline B1 & 2.75 & 3.61 & 1.86 & 0.32 & 1.16 \\
B2 & 5.86 & 5.42 & 2.79 & 1.79 & 6.37 \\
\hline
\end{tabular}

195

3.3. $T H G$ response study

197 The measurements of THG response in B1 and B2 films were performed for $s-s$ incident198 generated polarization scheme (Fig. 6). It can be seen that B2 film represents much higher THG 
response compared to B1 film. As the THG originates from the film together with the glass

200 substrate, the impact of substrate was taken into account during data processing and the cubic

201 NLO susceptibility calculation.

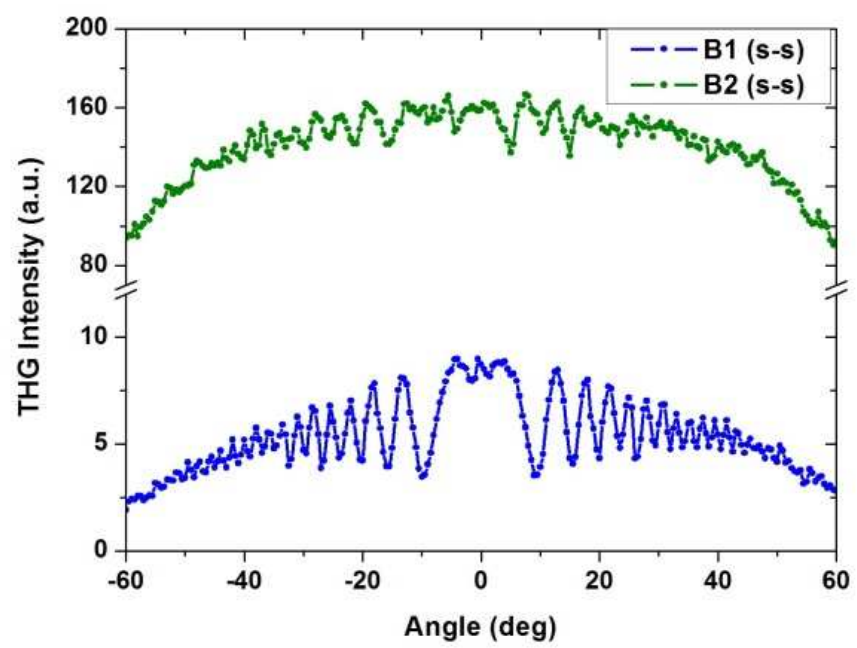

204 Fig. 6. THG intensities from B1 and B2 films as a function of incident angle for the $s-s$ 205 polarization of the fundamental beam - generated signal.

Third order NLO susceptibility was calculated using the comparative model which takes into

208 account linear optical absorption [36]:

$$
\chi^{(3)}=\chi_{\text {Silica }}^{(3)}\left(\frac{2}{\pi}\right)\left(\frac{l_{\text {Silica }}^{\text {coh }}}{d}\right)\left(\frac{\frac{\alpha d}{2}}{1-e^{-\frac{\alpha d}{2}}}\right)\left(\frac{I^{3 \omega}}{I_{\text {Silica }}^{3 \omega}}\right)^{1 / 2}
$$

212 where $\chi_{\text {Silica }}^{(3)}$ is the cubic susceptibility of silica, $l_{\text {Silica }}^{\text {coh }}$ is the coherent length of silica, $\alpha$ is the

213 absorption coefficient at triple laser wavelength, $d$ is the film thickness, $I^{3 \omega}$ and $I_{\text {Silica }}^{3 \omega}$ are the

214 THG intensities of the samples and silica at the same conditions, respectively. The calculated 
215 values of $\chi^{(3)}$ for the B1 and B2 films are given in Table 1 and they are one-to-two orders of

216 magnitude higher than for silica $\left(2 \cdot 10^{-20} \mathrm{~m}^{2} \mathrm{~V}^{-2}\right)$. The compound B2 is characterized with a much

217 higher third order NLO susceptibility than B1 due to more effective electron delocalization

218 caused by charge transfers from the dimethylamino groups to the BODIPY center together with

219 its D-A-D structure, contributing to the microscopic nonlinearity.

220 The electronic contribution of second hyperpolarizability $\gamma_{\text {elec }}$ for B1 and B2 molecules was

221 deducted from third order susceptibility (Table 1), as THG measurements are only dealing with

222 electronic constituent, taking into account the concentration of the molecule and the local field

223 factor [37, 38]. The comparatively higher $\gamma_{\text {elec }}$ for $\mathrm{B} 2$ is attributed to the presence of two

224 dimethylaminostyryl substituents which imparts a greater intramolecular charge-transfer

225 character compared to B1 compound. It is worth noting that except for the intra-molecular

226 electronic contribution some role may also play vibrational contribution to hyperpolarizability of

227 the systems.

\section{Conclusions}

In summary, B1 and B2 BODIPY-based derivatives with one and two conjugated

231 dimethylaminostyryl substituents exhibit considerable second and third order NLO responses at

$2321064 \mathrm{~nm}$, which can be tuned by the number of these peripheral substituents. In particular, the 233 expansion of the $\pi$-conjugated system by two electron donor groups, leading to a D-A-D 234 structure, results in significant increase of both SHG and THG responses comparing to the D-A 235 structure. Attaching strong electron-donating groups on the BODIPY core can thus promote 236 charge transfer character in these compounds and enhance their second and third order NLO 237 properties. Varying the electron donating efficiency and the respective position of donor and 
238 acceptor groups in BODIPY conjugated systems will be the object of further studies with the aim

239 to establish applicable structure-property relationships.

\section{Acknowledgements}

242 B. K. acknowledges the Pays de la Loire region for the financial support of research work in

243 the framework of the "Molecular Systems for Nonlinear Optical Application" (MOSNOA)

244 LUMOMAT project. S. T. acknowledges the MESRSFC, IFM, and CNRST of Morocco.

\section{Appendix A. Supplementary data}

Supplementary data related to this article can be found at http://dx.doi.org/

\section{References}

250 [1] Guzow K, Kornowska K, Wiczk W. Synthesis and Photophysical Properties of a New 251 Amino Acid Possessing a BODIPY Moiety. Tetrahedron Lett 2009; 50: 2908-10.

252 [2] Tram K, Yan H, Jenkins HA, Vassiliev S, Bruce D. The Synthesis and Crystal Structure of 253 Unsubstituted 4,4-difluoro-4-bora-3a,4a-diaza-s-indacene (BODIPY). Dyes Pigm 2009; 82: $254 \quad 392-5$.

255 [3] Arroyo IJ, Hu R, Merino G, Tang BZ, Pena-Cabrera E. The Smallest and One of the 256 Brightest. Efficient Preparation and Optical Description of the Parent Borondipyrromethene System. J Org Chem 2009; 74: 5719-22.

[4] Haugland RP. Handbook of fluorescent probes and research chemicals. 9th ed. Eugene: Molecular Probes Inc.; 2002. 
[5] Umezawa K, Nakamura Y, Makino H, Citterio D, Suzuki K. Bright, Color-Tunable Fluorescent Dyes in the Visible-Near-Infrared Region. J Am Chem Soc 2008; 130: 1550-51.

[6] Gabe Y, Urano Y, Kikuchi K, Kojima H, Nagano T. Highly Sensitive Fluorescence Probes for Nitric Oxide Based on Boron Dipyrromethene ChromophoreRational Design of Potentially Useful Bioimaging Fluorescence Probe. J Am Chem Soc 2004; 126: 3357-67.

[7] Qi X, Jun JE, Xu L, Kim SJ, Hong JSJ, Yoon YJ, Yoon JJ. New BODIPY Derivatives as OFF-ON Fluorescent Chemosensor and Fluorescent Chemodosimeter for $\mathrm{Cu}^{2+}$ : Cooperative

[8] Loudet A, Burgess K. BODIPY Dyes and Their Derivatives: Syntheses and Spectroscopic Properties. Chem Rev 2007; 107: 4891-932.

[9] Lee JS, Kang NY, Kim YK, Samanta A, Feng S, Kim HK, Vendrell M, Park JH, Chang YT. Synthesis of a BODIPY Library and Its Application to the Development of Live Cell Glucagon Imaging Probe. J Am Chem Soc 2009; 131: 10077-82.

[10] Shao J, Sun H, Guo H, Ji S, Zhao J, Wu W, Yuan X, Zhang C, James TD. A highly selective red-emitting FRET fluorescent molecular probe derived from BODIPY for the detection of cysteine and homocysteine: an experimental and theoretical study. Chem Sci 2012; 3: 104961.

[11]Duan X, Li P, Li P, Xie T, Yu F, Tang B. The Synthesis of Polarity-Sensitive Fluorescent Dyes Based on the BODIPY Chromophore. Dyes Pigm 2011; 89: 217-22.

[12] Li F, Yang SI, Ciringh YZ, Seth J, Martin CH, Singh DL, Kim DH, Birge RR, Bocian DF, Holten D, Lindsey JS. Design, Synthesis, and Photodynamics of Light-Harvesting Arrays Comprised of a Porphyrin and One, Two, or Eight Boron-Dipyrrin Accessory Pigments. J Am Chem Soc 1998; 120: 10001-17. 
283 [13] Wu W, Guo H, Wu W, Ji S, Zhao J. Organic Triplet Sensitizer Library Derived from a 284 Single Chromophore (BODIPY) with Long-Lived Triplet Excited State for Triplet-Triplet Annihilation Based Upconversion. J Org Chem 2011; 76: 7056-64.

[14] Mula S, Ray AK, Banerjee M, Chaudhuri T, Dasgupta K, Chattopadhyay S. Design and Development of a New Pyrromethene Dye with Improved Photostability and Lasing Efficiency: Theoretical Rationalization of Photophysical and Photochemical Properties. J Org Chem 2008; 73: 2146-54.

Demonstration of the Heavy-Atom Effect for Photodynamic Therapy. J Am Chem Soc 2004; 126: $10619-31$.

[16]Zhao J, Wu W, Sun J, Guo S. Triplet Photosensitizers: from Molecular Design to Applications. Chem Soc Rev 2013; 42: 5323-51.

[17] Bouit PA, Kamada K, Feneyrou P, Berginc G, Toupet L, Maury O, Andraud C. Two-Photon Absorption-Related Properties of Functionalized BODIPY Dyes in the Infrared Range up to Telecommunication Wavelengths. Adv Mater 2009; 21: 1151-4.

[18] Ulrich G, Barsella A, Boeglin A, Niu S, Ziessel R. BODIPY-Bridged Push-Pull Chromophores for Nonlinear Optical Applications. ChemPhysChem 2014; 15: 2693-700.

[19] Shi WJ, Lo PC, Singh A, Ledoux-Rak I, Ng DKP. Synthesis and Second-Order Nonlinear Optical Properties of Push-Pull BODIPY Derivatives. Tetrahedron 2012; 68: 8712-8.

[20] Wang Y, Zhang D, Zhou H, Ding J, Chen Q, Xiao Y, Qian S. Nonlinear Optical Properties and Ultrafast Dynamics of Three Novel Boradiazaindacene Derivatives. J Appl Phys 2010; 108: 033520-8. 
[21]Zhang D, Wang Y, Xiao Y, Qian S, Qian X. Long-Wavelength Boradiazaindacene Derivatives with Two-Photon Absorption Activity and Strong Emission: Versatile Candidates for Biological Imaging Applications. Tetrahedron 2009; 65: 8099-103.

[22] Potamianos D, Giannakopoulou P, Kaloudi-Chantzea A, Pistolis G, Couris, S. Third-Order Nonlinear Optical Properties of Some Novel BODIPYs. Proceedings of the 16th International Conference on Transparent Optical Networks (ICTON). Graz: IEEE; 2014.

[23]Zhu M, Jiang L, Yuan M, Liu X, Ouyang C, Zheng H, Yin X, Zuo Z, Liu H, Li Y. Efficient Tuning Nonlinear Optical Properties: Synthesis and Characterization of a Series of Novel Poly(aryleneethynylene)s Co-Containing BODIPY. J Polym Sci A Polym Chem 2008; 46: 7401-10.

[24] Kim B, Yue X, Sui B, Zhang X, Xiao Y, Bondar MV, Sawada J, Komatsu M, Belfield KD. Near-Infrared Fluorescent 4,4-Difluoro-4-bora-3a,4a-diaza-s-indacene Probes for One- and Two-Photon Fluorescence Bio-imaging. Eur J Org Chem 2015; 2015: 5563-71.

[25]Zhang X, Xiao Y, Qi J, Qu J, Kim B, Yue X, Belfield KD. Long-Wavelength, Photostable, Two-Photon Excitable BODIPY Fluorophores Readily Modifiable for Molecular Probes. J Org Chem 2013; 78: 9153-60.

[26] Zhao Z, Chen B, Geng J, Chang Z, Aparicio-Ixta L, Nie H, Goh CC, Ng LG, Qin A, RamosOrtiz G, Liu B, Tang BZ. Red Emissive Biocompatible Nanoparticles from Tetraphenylethene-Decorated BODIPY Luminogens for Two-Photon Excited Fluorescence Cellular Imaging and Mouse Brain Blood Vascular Visualization. Part Part Syst Char 2014; 31: 481-91.

[27] Kulyk B, Taboukhat S, Akdas-Kilig H, Fillaut J-L, Boughaleb Y, Sahraoui B. Nonlinear refraction and absorption activity of dimethylaminostyryl substituted BODIPY dyes. RSC Adv 2016; 6: 84854-9. 
329 [28] Rurack K, Kollmannsberger M, Daub J. Molecular Switching in the Near Infrared (NIR)

330 with a Functionalized Boron-Dipyrromethene Dye. Angew Chem Int Ed 2001; 40: 385-7.

331 [29] Deniz E, Isbasar GC, Bozdemir ÖA, Yildirim LT, Siemiarczuk A, Akkaya EU. Bidirectional 332 Switching of Near IR Emitting Boradiazaindacene Fluorophores. Org Lett 2008; 10: 3401-3.

333 [30] Maker PD, Terhune RW, Nisenhoff M, Savage CM. Effects of Dispersion and Focusing on 334 the Production of Optical Harmonics. Phys Rev Lett 1962; 8: 21-2.

335 [31] Kulyk B, Kerasidou AP, Soumahoro L, Moussallem C, Gohier F, Frère P, Sahraoui B. 336 Optimization and Diagnostic of Nonlinear Optical Features of $\pi$-conjugated BenzodifuranBased Derivatives. RSC Adv 2016; 6: 14439-47.

[32] Schmitt A, Hinkeldey B, Wild M, Jung G. Synthesis of the Core Compound of the BODIPY Dye Class: 4,4'-Difluoro-4-bora-(3a,4a)-diaza-s-indacene. J Fluoresc 2009; 19: 755-8.

[33] Cho DW, Fujitsuka M, Ryu JH, Lee MH, Kim HK, Majima T, Ima C. S2 Emission from Chemically Modified BODIPYs. Chem Commun 2012; 48: 3424-6.

[34]Lee GJ, Cha SW, Jeon SJ, Jin J-I, Yoon JS. Second-Order Nonlinear Optical Properties of Unpoled Bent Molecules in Powder and in Vacuum-Deposited Film. J Korean Phys Soc

345 [35] El Ouazzani H, Iliopoulos K, Pranaitis M, Krupka O, Smokal V, Kolendo A, Sahraoui B. 346 Second- and Third-Order Nonlinearities of Novel Push-Pull Azobenzene Polymers. J Phys $347 \quad$ Chem B 2011; 115: 1944-9.

348 [36] Kubodera K, Kobayashi H. Determination of Third-Order Nonlinear Optical Susceptibilities for Organic Materials by Third-Harmonic Generation. Mol Cryst Liq Cryst 1990; 182: 103-

351 [37] Boyd RW. Nonlinear Optics. 2nd ed. Boston, Amsterdam: Academic Press; 2003. 
[38] Stegeman GI, Stegeman RA. Nonlinear optics: phenomena, materials, and devices. Hoboken:

353 Wiley; 2012. 
The nonlinear optical properties of functionalized BODIPY models were studied.

The nonlinearities are dependent on the number of dimethylaminostyryl substituents.

Electron-donating groups enhance second and third order NLO response of BODIPY. 